\title{
Lasers are used in Nanostructuring, Microarray Analysis and Surgery
}

\section{Sharma KR*}

Department of Physics, College of Science and Technology, Texas Southern University, Houston, TX 77446

\section{Editorial}

Lasers are used in nanostructuring operations that are depositional, epitaxial and lithographical in nature, in surgery, confocal scanners in microarray analysis and next-generation sequencing, and number of research applications. Atoms can be trapped using lasers and affect lower temperatures. Laser cooling may lead to agglomeration of atoms. Chu and Philips obtained the Nobel Prize in 1997. Nanoprisms can be generated using laser ablation techniques. The market for nanotechnology products is expected to exceed 1 trillion later this year. The break-down by industry type are about $\$ 340$ billion from nanomaterials, $\$ 300$ billion from electronics, $\$ 180$ billion from pharmaceuticals, $\$ 100$ billion each form chemicals and sustainability and $\$ 70$ billion from transportation. Genominomics cost has decreased dramatically over the past 10 years. This may lead to a wider use of genome sequencers. Confocal scanners are used during microarray analysis. Cost of lasers may also be driven down by increased demand. Materials that went into the first semiconductor lasers can be used to make miniature lasers that are lower in cost, smaller in size. Alferov and Kromer were given the Nobel Prize in 2000 for their development of semiconductor physics. Both light source and energy supply are placed in mirrors in one mm sized crystals.

The origins of lasers can be traced back to the writings of Sir Albert Einstein on the theory of stimulated emission in 1917. Holography was theorized by Nobel laureate Gabor in 1947. Holograms with their three dimensional images was portrayed in crime novels to confuse the witness between the criminal and image. Ruby laser that generated strong pulses of red light was operational in 1960. Semiconductor lasers came about in 1963 and Alferov and Kromer obtained the Physics Nobel Prize in 2000. Corning glass introduced optical fibers with lower losses and was used in telecommunications over longer distances. Laser spectroscopy was developed and Bloembergen and Schawlow obtained the Nobel Prize in 1981[1].

Pulses of laser radiation are used to evaporate material from a target, which is then deposited on a substrate in order to produce thin films in the Pulsed laser deposition (PLD) technique. This technique is used in research to study the change of thermal conduction with temperature in aluminum and change in electrical conduction in the same material over the same temperature range. Aluminum showed increase in thermal conductivity for increases in temperature and was flat for electrical conductivity as a function of temperature. Laser beams are easier to transport and manipulate and have larger dynamic ranges of delivered energy compared with other beams. PLD can be used to achieve 100 times higher deposition rates compared with other epitaxial methods. Particles are created during PLD process. So its usage in the semiconductor industry to generate high quality films in limited. Nanosecond laser pulses with low repetition rates have been found to generate film with a surface free of particles and with improved surface quality. Energy from each pulse of laser is not sufficient to evaporate the target material. This results in particle deposits on the substrate used in order to form the desired film. Particles are eliminated from the vapor plume. Thermodynamic parameters of the target are used in the selection of energy of the laser pulse and optimal laser intensity. This results in improved efficiency of the evaporation process. Ultrafast laser ablation at a succession of short laser pulses at high repetition rate are used for heating the target and generating successive bursts of atoms and ions in a vapor plume. This results in deposition of thin structural films that are amorphous in character. A condition where a continuous beam of atom strikes the substrate can be reached by increasing the laser repetition rate. The spread of atom velocities in the laser-evaporated plume allows the slow atoms from one pulse to arrive at the same time as the faster atoms from later pulses. As a result, the film grows on the substrate surface from a continuous flow of atomic vapors with regulated atom flux density. Epitaxial growth can be realized. The relaxation times for carbon vapor can be estimated at 1.1 milliseconds [2].

When the duration time of the pulse is decreased from nanoseconds to picoseconds, the evaporation process takes place in a narrower zone near the target surface. There is insufficient time for thermal energy transport further into the target during thelaser pulse. Almost all the absorbed laser energy goes into evaporation of atoms from a very thin zone near the target surface. Less waste heat is generated from absorbed laser energy. A 10-ns pulse increased to a 100 -ps pulse results in a 10 times decrease of the energy density needed to ablate the material, assuming that the laser evaporation is at the optimal regime in bothcases. The advantage of using short laser pulses is the elimination of particles and droplets in the vapor flow. There is a dramatic increasein the number of atoms evaporated by a single pulse. Typically conventional PLD in optimal conditions leads to the evaporation of $10^{19}$ atoms/pulse, which is sufficient to generate micron-sized particles. The patented method uses very low energy pulses on $10^{11}$ atoms perpulse, which leads to elimination of particles in the vapor flow. This leads to better surface quality films.

Nan clusters have a major impact on a spectrum of applications, including chemical and bio detection, catalysis, optics, and data storage. These nanostructures are typically made from molecular precursors, and there are now a wide variety of compositions, sizes, and even shapes available. Because of their unusual and potentially useful optical properties, nanoprism structures in particular have been a recent synthetic target of many researchers. A high-yield photosynthetic method for the preparation of triangular nanoprisms from silver nanospheres was recently reported. Elementary particles that were confirmed using the Hadron collider experiments in 2011 were triangular prismatic in structure. These experiments have been conducted 100 years after the Rutherfold's experiments that were used in order to confirm the presence of the nucleus in the atom.

*Corresponding author: Sharma KR, Department of Physics, College of Science and Technology, Texas Southern University, Houston, USA, Tel: +281-256-2976; E-mail: jyoti_kalpika@yahoo.com

Received July 10, 2015; Accepted July 10, 2015; Published July 12, 2015

Citation: Sharma KR (2015) Lasers are used in Nanostructuring, Microarray Analysis and Surgery. J Laser Opt Photonics 2: e105. doi:10.4172/jlop.1000e105

Copyright: () 2015 Sharma KR. This is an open-access article distributed under the terms of the Creative Commons Attribution License, which permits unrestricted use, distribution, and reproduction in any medium, provided the original author and source are credited. 
For many nanoparticle syntheses, an Ostwald ripening mechanism,where large clusters grow at the expense of smaller ones, is used to describe and model the growth processes. This type of ripening typically results in unimodal particle growth. Thus, a method of controlling the growth and ultimate dimensions of such structures is desired. Such a method will be different from the Ostwald ripening mechanisms.

A method of formation of Nanoprisms by exposing silver particles toa wavelength of light between approximately 400 and $700 \mathrm{~nm}$ for aperiod of less than approximately $60 \mathrm{~h}$ was proposed. The nanoprismsformed have a bimodal size distribution. The silver particles are present in a colloid containing a reducing agent, a stabilizing agent, and a surfactant. If the colloid contains a stabilizing agent anda surfactant, the ratio of the stabilizing agent to the surfactant is preferably about $0.3: 1$. The nanoparticle starting materials have a diameter between $0.2 \mathrm{~nm}$ and approximately $15 \mathrm{~nm}$. The nanoprisms formedare single crystalline and have a $\{111\}$ crystal face on a base plane of the nanoprism and a $\{110\}$ crystal face on a side plane of the nanoprism and display plasmon bands having $\lambda_{\max }$ at 640 and $1065 \mathrm{~nm}$, and 340 and $470 \mathrm{~nm}$, respectively [3].

Treatment of glaucoma by direct alternation of the optic nerve. They are also used in weight loss program. Models also find use for them. The confocal scanners used in microarray analysis call for lasers to provide the excitation beam [4].

\section{References}

1. http://nobel.se

2. Sharma KR (2005) Damped wave transport and relaxation, Elsevier, Amsterdam.

3. Sharma KR (2010) Nanostructuring operations in nanoscale science and engineering, McGraw Hill Professional, New York.

4. Sharma KR (2015) Microarray analysis: Biochips and eradication of al diseases, Momentum Press, New York. 